\title{
Simultaneous Adsorption of Ni(II) Ion and Anionic Dyes by a Novel Silica Gel Functionalized with Amino-EDTA
}

\author{
Jinli FANG ${ }^{1,2, a}$, Zhijun XU ${ }^{1,2, b}$, Yunfeng $\mathrm{HUANG}^{2, \mathrm{c}}$, Zhichao LIN ${ }^{2, \mathrm{~d}}$, Qiang \\ WANG $^{1,2, e, *}$
}

${ }^{1}$ Engineering Research Center for Clean Production of Textile Dyeing and Printing, Ministry of Education, Wuhan Textile University, Wuhan 430073, China

${ }^{2}$ School of Chemistry and Chemical Engineering, Wuhan Textile University, Wuhan 430073, China

${ }^{*}$ Corresponding author

aemail: 827898083@qq.com, bemail: xzjwtu@163.com, 'email: 984701284@qq.com, demail:493687180@qq.com, eemail: qiang_wang@wtu.edu.cn

Keywords: Functionalized Silica Gel; Adsorption; Nickel Ion; Anionic Dyes Removal

\begin{abstract}
Amino-EDTA functionalized silica gel was prepared, and it was used to absorb Ni(II) ion from aqueous solution to form SG-Ni, via the coordination interaction of $\mathrm{Ni}$ (II) ion with EDTA group on the surface of the silica gel. The adsorption of anionic dyes including Acid Orange II (AO II) and Reactive Brilliant Red X-3B (X-3B) in aqueous solution with SG-Ni were investigated in batch techniques. The effect of time, dye concentration, and dosage of the adsorbent were studied. The adsorption equilibrium data of both dyes were fitted better to Langmuir adsorption model and kinetics studies of both dyes revealed that the adsorption processes followed a pseudo second-order model.
\end{abstract}

\section{Introduction}

Purification of industrial effluents loaded with heavy metal ions is one of the major problems on wastewater treatment because of their toxicity and they cannot be degraded by means of chemical or biological ways in nature [1]. The heavy ions can be accumulated and transferred into organisms by the food chain, so they do harm to environment and human health even at relatively low concentrations. It is noted that heavy metal ions are frequently coexistent with dyes in numerous industrial waste waters [2]. It is known to us that most of dyes are toxic and potentially carcinogenic to human beings. Therefore, it is important to remove the toxic metals and dyes from wastewaters before being discharged into the environment.

Adsorption technique because of its effectiveness, flexibility, economy, and simplicity in design, ease of operation with no unwanted side products has been widely used in the removal of dyes, organic pollutants, and heavy metal ions from wastewater [3]. Silica gel contains a large number of silanol groups $(\mathrm{SiOH})$ on the surface, which enable the grafting of organic functional groups, has been one of the most popular adsorbents. Chemical modification of silica with chelating agents such as EDTA which form very strong complex with metal ions may produce adsorbents with high adsorption capacity and short equilibrium time [4].

In this paper, amino-EDTA functionalized silica gel was prepared and the research of simultaneous removal of metal ions and anionic dyes was studied. The kinetic and thermodynamic parameters of adsorption for two anionic dyes were analyzed.

\section{Experimental}

\section{Materials.}

Silica gel with particle size in the range of 100-200 mesh was purchased from Qingdao Ocean Chemical Company, China. Ethylenediaminetetraacetic acid (EDTA), N, N'-Diisopropylcarbodiimide (DIC) and N-[3-(Trimethoxysilyl) propyl] ethylenediamine (95\%) were obtained from Aladdin Reagent Co. Ltd, China. N, N-Dimethylformamide (DMF), Nickel nitrate hexahydrate 
( $\mathrm{NiSO}_{4} \cdot 6 \mathrm{H}_{2} \mathrm{O}$ ), Toluene, Methanol, Ether were purchased from Sinopharm Chemical Reagents Co. Ltd, China. These chemicals and reagents were used as supplied without further purification. Dry toluene was distilled under $\mathrm{N}_{2}$ from $\mathrm{CaH}_{2}$ and dry methanol from magnesium methoxide. All aqueous solutions for measurement experiments were prepared by using doubly distilled water, and the other reagents were of analytical grade (AR).

Preparation of functionalized silica gel and its adsorption for Ni(II) ions and anionic dyes.

Silica gel was pretreated [5] and modified with N-[3-(Trimethoxysilyl)propyl]ethylenediamine, following grafted with ethylenediaminetetraacetic acid (EDTA). The adsorption of amino-EDTA functionalized silica gel for $\mathrm{Ni}(\mathrm{II})$ was performed by adding the adsorbent in $\mathrm{NiSO}_{4}$ solution. The yellowish silica gel turned to light green shortly, suggesting the fast adsorption of $\mathrm{Ni}$ (II) ion onto the surface of silica gel forming Nickel(II) bound silica gel, SG-Ni. The SG-Ni was added into the Acid Orange II (AO II) and Brilliant Red X-3B (X-3B) solution, respectively, and the mixture was shaken at room temperature for 3 hours. The dye adsorption percentages were calculated based on the absorbance $\left(\lambda_{\max }=484 \mathrm{~nm}\right.$ for AO II or $\lambda_{\max }=538 \mathrm{~nm}$ for X-3B) of the residue dye solution. Above processes are described in Figure 1.

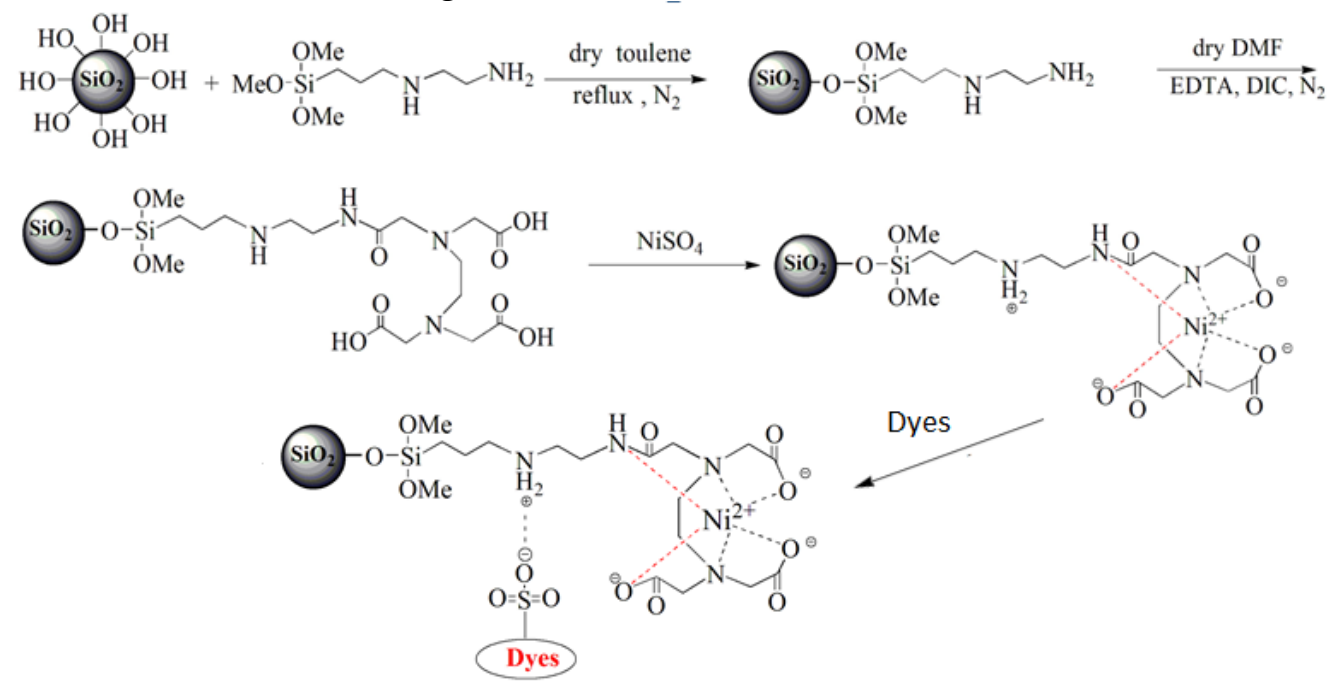

Fig.1. Preparation of functionalized silica gel and its adsorption of nickel ions and anionic dyes

\section{Results and Discussion}

\section{Effects of contact time.}

The effects of contact time on the removal of Anionic dyes by the SG-Ni are shown in Figure 2. The removal of dyes by SG-Ni was rapid in the initial stages of adsorption and decreased with time until equilibrium. Initial adsorption was fast due to the many vacant adsorption sites.

Effects of dye concentration.

The effect of initial dye concentration on anionic dyes removal was studied in the concentration ranges from 20 to $200 \mathrm{mg} \mathrm{L}^{-1}$ as shown in Figure 3. It indicates that the adsorption capacity (qe) of the SG-Ni increases with increasing of dye concentration, and reaches to maximum value at certain concentration ranges.

\section{Effects of absorbent dosage.}

The effects of absorbent dosage on the removal of anionic dyes are shown in Figure 4. It was investigated by contacting AO II solution or X-3B solution with different amounts of SG-Ni. The results reveal that the dye removal rate increases when the absorbent dosage increases, since the higher dosage absorbent supply more number of active sites for the interaction with anionic dyes. 

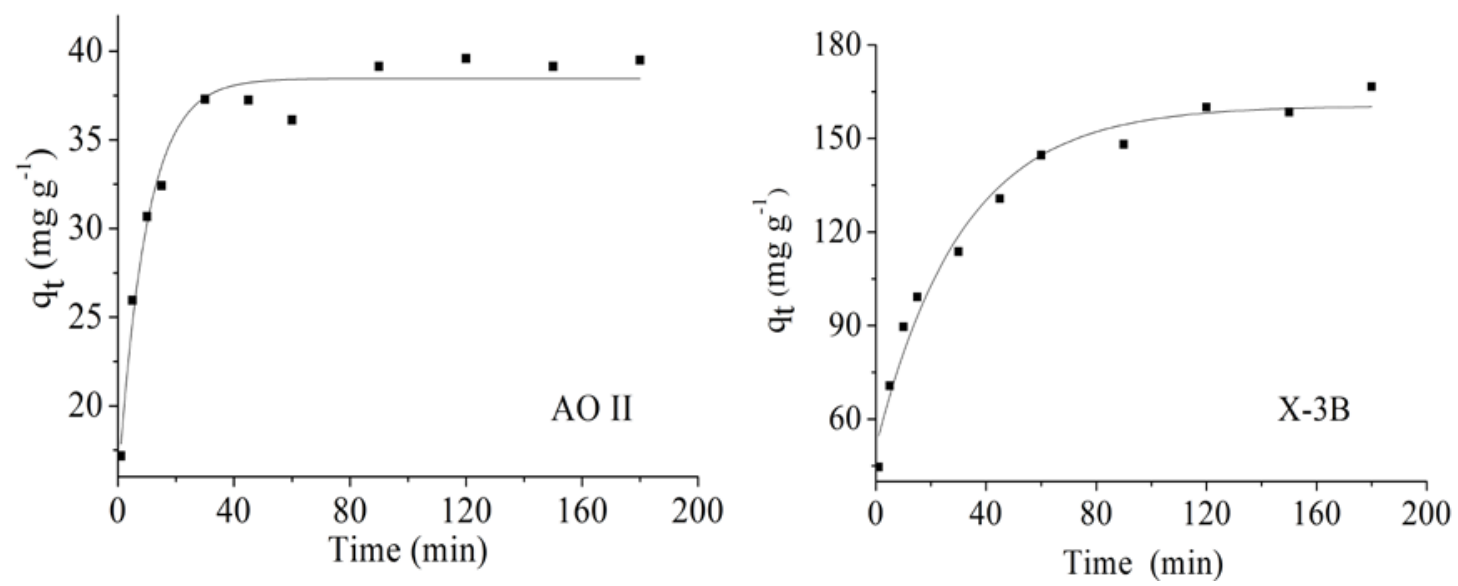

Fig.2. Effect of contact time on the removal of Anionic dyes by SG-Ni
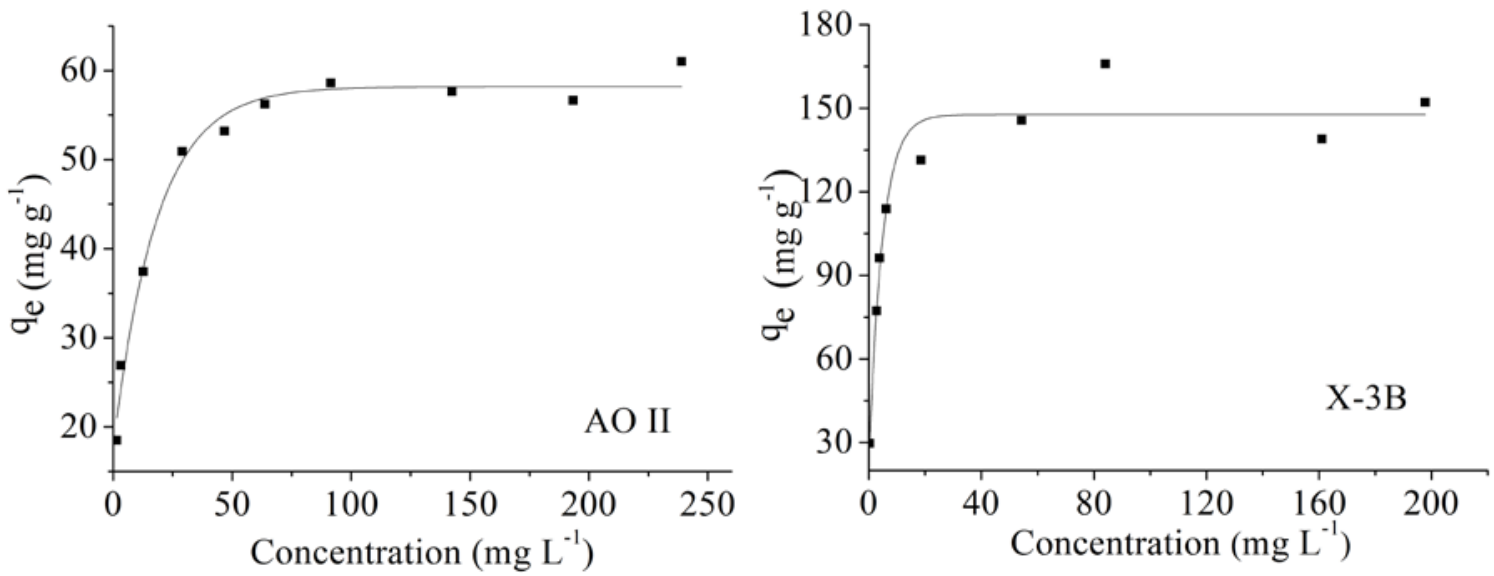

Fig.3. Effect of dye concentration on the removal of anionic dyes by SG-Ni
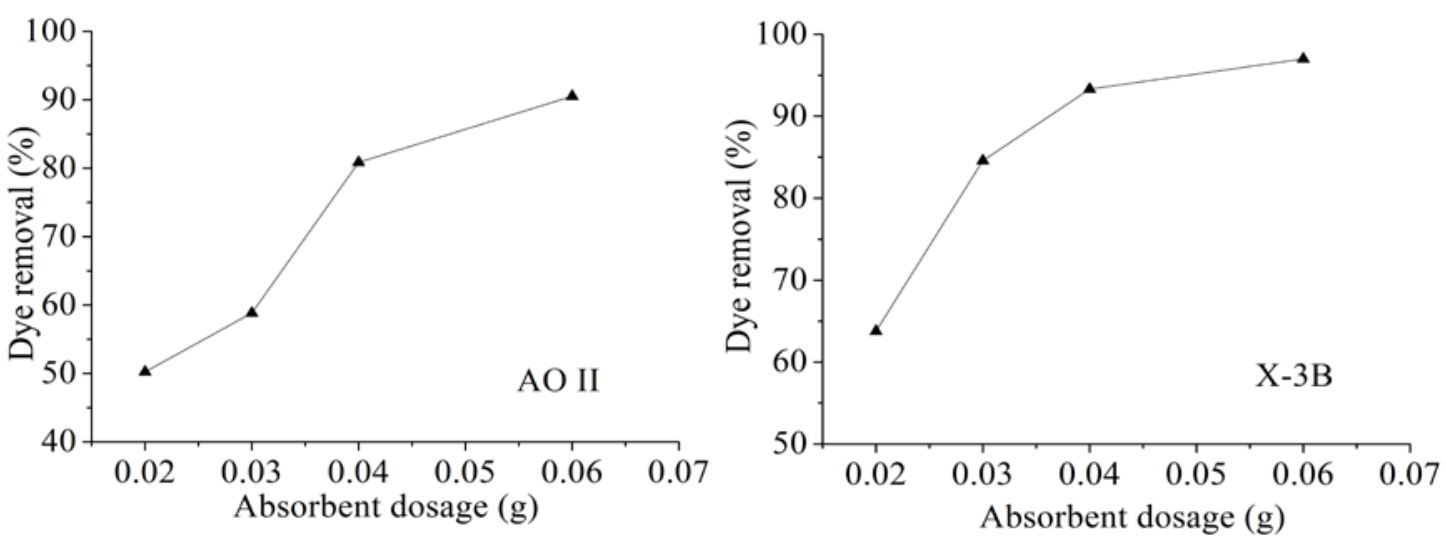

Fig.4. Effect of absorbent dosage on the removal of anionic dyes by SG-Ni

\section{Adsorption kinetics.}

Empirically obtained kinetic adsorption data for AO II and X-3B on SG-Ni were fitted to the pseudo-first-order and pseudo second-order models shown in Eqs. (1) and (2), respectively [6], and the results are listed in Table 1 . The parameters of $k_{1}$ and $k_{2}$ are the pseudo first-order and second-order rate constant $\left(\mathrm{min}^{-1}\right)$ of adsorption; qe (Cal.) $\left(\mathrm{mg} \mathrm{g}^{-1}\right)$ values are the amounts of dye adsorbed per unit mass of absorbent at equilibrium. The value of pseudo first-order and second-order rate constant and regression coefficient $\left(R^{2}\right)$ values are also in Table 1 . The results show that the correlation coefficients $\left(R^{2}\right)$ of the pseudo-second-order kinetic model $\left(\mathrm{R}^{2}>0.99\right)$ are much higher than that of the pseudo-first-order model. In addition, the experimental values of $q_{\mathrm{e}}$ (Exp.) are very similar to the values calculated by the pseudo-second-order equation $q_{\mathrm{e}}\left(q_{\mathrm{e}}(\mathrm{Cal}).\right)$. 
Thus, the adsorption can be better described by the pseudo-second-order kinetic model rather than the pseudo-first-order kinetic model.

$$
\begin{aligned}
& \ln \left(q_{e}-q_{t}\right)=\ln q_{e}-k_{1} t \\
& \frac{t}{q_{t}}=\frac{1}{k_{2} q_{e}^{2}}+\frac{t}{q_{e}}
\end{aligned}
$$

\begin{tabular}{|c|c|c|c|c|c|c|c|}
\hline \multirow[b]{2}{*}{ Dye } & \multirow[b]{2}{*}{$\begin{array}{r}q_{e}(\text { Exp. }) \\
\left(\mathrm{mg} \mathrm{g}^{-1}\right)\end{array}$} & \multicolumn{3}{|c|}{ Pseudo-first-order } & \multicolumn{3}{|c|}{ Pseudo-second-order } \\
\hline & & $\begin{array}{c}k_{1} \\
\left(\min ^{-1}\right)\end{array}$ & $\begin{array}{c}q_{e(C a l .)} \\
\left(\mathrm{mg} \mathrm{g}^{-1}\right)\end{array}$ & $R^{2}$ & $\begin{array}{c}k_{2} \\
\left(\mathrm{~g} \mathrm{mg}^{-1} \min ^{-1}\right)\end{array}$ & $\begin{array}{r}q_{e}(\mathrm{Cal}) \\
\left(\mathrm{mg} \mathrm{g}^{-1}\right)\end{array}$ & $R^{2}$ \\
\hline AO II & 38.45 & 0.04235 & 12.47 & 0.6118 & 0.0083557 & 40.08 & 0.9990 \\
\hline$X-3 B$ & 160.16 & 0.03848 & 130.81 & 0.8760 & 0.0005847 & 171.23 & 0.9960 \\
\hline
\end{tabular}

Table 1. Kinetic model parameters for the adsorption of AO II and X-3B on SG-Ni

\section{Adsorption thermodynamics.}

The parameters of the Langmuir (3) and Freundlich (4) models along with the regression coefficients $\left(R^{2}\right)$ are listed in Table 2. The $R^{2}$ values in Table 2 reveal that the Langmuir isotherm fits the experimental results better than the Freundlich model, and it suggests that the adsorption of AO II and X-3B onto SG-Ni follows the mechanism of monolayer adsorption (chemisorption) on a homogenous surface [5].

$$
\begin{aligned}
& \frac{C_{e}}{q_{e}}=\frac{C_{e}}{q}+\frac{1}{q K_{L}} \\
& \ln q_{e}=\ln K_{F}+\frac{\ln C_{e}}{n}
\end{aligned}
$$

Table 2 The isotherm parameters for the dyes adsorption onto SG-Ni

\begin{tabular}{lllcccc}
\hline Dye & \multicolumn{3}{c}{ Langmuir } & \multicolumn{5}{c}{ Freundlich } \\
\hline & $q\left(\mathrm{mg} \mathrm{g}^{-1}\right)$ & $k_{L}\left(\mathrm{~L} \mathrm{mg}^{-1}\right)$ & $R^{2}$ & $k_{F}\left(\mathrm{mg} \mathrm{g}^{-1}\right)$ & $n$ & $R^{2}$ \\
AO II & 60.64 & 0.1828 & 0.9980 & 20.330 & 4.499 & 0.9020 \\
X-3B & 149.48 & 0.7568 & 0.9930 & 57.971 & 4.529 & 0.7920 \\
\hline
\end{tabular}

\section{Conclusions}

It is evident that the amino-EDTA modified silica gel was effective for Ni(II) ion and anionic dyes removal from aqueous solution. Its adsorption capacity for anionic dyes increased with increasing of time, initial concentration of the dye solution, and the SG-Ni dosage. The maximum dye adsorption capacity of SG-Ni for AO II and X-3B was $60.64 \mathrm{mg} \mathrm{g}^{-1}$ and $149.48 \mathrm{mg} \mathrm{g}^{-1}$, respectively. The adsorption processes of the AO II and X-3B on SG-Ni followed a pseudo second-order model and experimental data fitted well with Langmuir adsorption model. The results suggest that the amino-EDTA modified silica gel maybe a useful prototype material for the removal of heavy metals and anionic dyes from aqueous system.

\section{Acknowledgement}

In this paper, the research was sponsored by the National Nature Science Foundation of China (Project No. 21277106).

\section{References}

[1] M. Li, C. Feng, Q. Zeng. Preparation and characterization of multi-carboxyl-functionalized silica gel for removal of $\mathrm{Cu}$ (II), Cd (II), Ni (II) and Zn (II) from aqueous solution [J]. Applied Surface Science, 2014, 314:1063-1069.

[2] F. Fu, Q. Xuan, R. Chen, Y. Xiong. Removal of $\mathrm{Cu}^{2+}$ and dye from wastewater using the heavy 
metal precipitant n,n-bis-(dithiocarboxy) piperazine [J]. Environmental Chemistry Letters, 2006, 4:41-44.

[3] M.T. Yagub, T.K. Sen, S. Afroze, H.M. Ang. Dye and its removal from aqueous solution by adsorption: A review [J]. Advance in Colloid and Interface Science, 2014, 209:172-184.

[4] R. Kumar, M. Barakat, Y. Daza, H. Woodcock, J.Kuhn. EDTA functionalized silica for removal of $\mathrm{Cu}(\mathrm{II}), \mathrm{Zn}(\mathrm{II})$ and $\mathrm{Ni}(\mathrm{II})$ from aqueous solution [J]. Journal of Colloid and Interface Science, 2013, 408:200-205.

[5] Q. Wang, W. Gao, Y. Liu, J. Yuan, Z. Xu, Q. Zeng, Y. Li, M. Schröder. Simultaneous adsorption of $\mathrm{Cu}(\mathrm{II})$ and $\mathrm{SO}_{4}{ }^{2-}$ ions by a novel silica gel functionalized with a ditopic zwitterionic Schiff base ligand [J]. Chemical Engineering Journal, 2014, 250:55-65.

[6] Y. Cai, Y. Huang, F. Liu, L. He, L. Lin, Q. Zeng. Liquid ammonia dyeing of cationic ramie yarn with triazinyl reactive dyes [J]. Cellulose, 2014, 21:3841-3849. 